Proceedings of the

International Geometry Center

Vol. 13, no. 3 (2020) pp. 58-63

\title{
Open finite-to-one functions on open topological graphs
}

\author{
Igor Yu. Vlasenko
}

\begin{abstract}
The paper describes homotopy classes of open continuous functions on finite open topological graphs.
\end{abstract}

Анотація. В роботі описано класи гомотопії неперервних відкритих функцій на відкритих топологічних графах.

\section{INTRODUCTION}

By a topological graph we will mean a finite 1-dimensional CW-complex. Its 0-cells are called vertices and 1-cell are edges. We can always replace each vertex of degree 2 and a pair of edges incident to it with a single edge and assume that a topological graph has no vertices of degree 2 .

Denote by $\Sigma$ the class of all topological graphs. A topological space $G$ is called an open topological graph if there is a topological graph $\bar{G}$ such that $G$ is a complement to some set of vertices of degree $1 \mathrm{in} \bar{G}$. The corresponding topological graph $\bar{G}$ is called a closure of $G$. An edge of $G$ incident to a vertex of degree 1 will be called semifree. We will also regard the real line $\mathbb{R}$ as a special open topological graph with 1 edge and without vertices.

Let $f: X \rightarrow Y$ be a continuous map between topological spaces. Then $f$ is called open if the image of each open set is open. A map $f$ is open at a point $x \in G$ if there exists an open base of topology $B$ at $x$ such that for every neighborhood $U$ of $x$ in $B$ its image $f(U)$ is open. It is easy to see that $f$ is open iff it is open at each point $x \in X$.

2010 Mathematics Subject Classification: 54C10

UDC 515.126.2

Keywords: open map, continuous function, homotopy classes, open topological graphs

Ключові слова: відкрите відображення, неперервна функція, класи гомотопії, відкритий топологічний граф

DOI: http://dx.doi.org/10.15673/tmgc.v13i3.1880 
Recall also that $f$ is called finite-to-one whenever the preimage of each point is finite, [1].

\section{FUnCTIONS ON OPEN TOPOLOGICAL GRAPHS}

Lemma 2.1. Let $G$ be a topological graph without isolated vertices and $f: G \rightarrow \mathbb{R}$ a continuous function. Then $f$ is an open map iff the following two conditions hold:

(a) $f$ is strictly monotone on edges;

(b) for every vertex $v$ the open edges incident to it split into two nonempty collections: $A=\left\{e_{1}, \ldots, e_{k}\right\}$ and $B=\left\{e_{k+1}, \ldots e_{l}\right\}$ such that $f(A) \subset(-\infty, f(v))$ and $f(B) \subset(f(v),+\infty)$.

Proof. Necessity. (a) Suppose $f$ is an open map. Notice that then $f$ has no local extremes. Indeed, if $x \in G$ for example is a local maximum, then for a small neighborhood $U$ of $x$ we have that $f(U) \subset(-\infty, f(x)]$, and therefore $f(U)$ is not an open neighborhood of $f(x)$, which contradicts to the openness of $f$.

Assume that $f$ is not monotone on an edge $e$, so there are points $x \neq y \in e$ such that $f(x)=f(y)$. Let $[x, y] \subset e$ be the closed segment between $x$ and $y$, and $(x, y)$ be its interior. Then $f([x, y])$ is a compact connected subset of $\mathbb{R}$, and therefore it is either a point or a closed segment. If $f([x, y])$ is a point, then $f((x, y))$ is also a point and it not an open subset of $\mathbb{R}$, which contradicts to the openness of $f$. Otherwise, $f([x, y])$ is a segment of the form $[f(x), a]$ or $[a, f(x)]$ for some $a \in \mathbb{R}$, so there exists $z \in(x, y)$ with $f(z)=a$. But then $z$ is a local extreme of the restriction of $f$ to open set $(x, y)$, which again contradicts to the openness of $f$. This proves (a).

(b) Notice that it follows from (a) that if $e$ is an edge incident to some vertex $v$, then either $f(e) \subset(-\infty, f(v))$ or $f(e) \subset(f(v),+\infty)$.

Suppose condition (b) fails, so there exists a vertex such that for all edges $\left\{e_{i}\right\}$ incident to $v$ we have that

- either $f\left(\cup_{i} e_{i}\right) \subset(-\infty, f(v))$

- or $f\left(\cup_{i} e_{i}\right) \subset(f(v),+\infty)$.

Then $v$ is a local extreme of $f$, which contradicts to the opennes of $f$.

Sufficiency. Suppose (a) and (b) hold. Then (a) implies that $f$ is open at points of edges, while (b) implies openness of $f$ at vertices of $G$.

Corollary 2.2. If $G$ is a finite topological graph, then every open function $f: G \rightarrow \mathbb{R}$ is finite-to-one. 


\section{Existence of functions from the Class $\mathfrak{F}(G, \mathbb{R})$}

Denote by $\Theta$ the class of all connected open topological graphs.

For $G_{1}, G_{2} \in \Theta$ let $\mathfrak{F}\left(G_{1}, G_{2}\right)$ be the set of continuous open (and therefore finite-to-one) maps from $G_{1}$ to $G_{2}$. Such maps a used in particular for modelling of holomorphic maps of Riemann surfaces, e.g. [3].

The class $\mathfrak{F}(G, \mathbb{R})$ can be empty for some graphs in $\Theta$. The simplest examples of a graph $G \in \Theta$ such that the class $\mathfrak{F}(G, \mathbb{R})$ is empty are the circle or a graph that has only a vertex and one semifree edge.

Let $G \in \Theta$ and let $\bar{G} \in \Sigma$ be a closure of $G$. Denote by $\mathfrak{M}(\bar{G}, \mathbb{R} \cup\{ \pm \infty\})$ the class of functions on $\bar{G}$ which are strictly monotone on edges and have no local extremes at vertices of degree $>1$.

By Lemma 2.1 for every function $\bar{f} \in \mathfrak{M}(\bar{G}, \mathbb{R} \cup\{ \pm \infty\})$ its restriction to $G$ is a function of the class $\mathfrak{F}(G, \mathbb{R})$. Conversely, if $f$ belongs to $\mathfrak{F}(G, \mathbb{R})$, then $f$ extends to a unique continuous function $\bar{f} \in \mathfrak{M}(\bar{G}, \mathbb{R} \cup\{ \pm \infty\})$. In other words we have a canonical bijection:

$$
\mathfrak{M}(\bar{G}, \mathbb{R} \cup\{ \pm \infty\}) \rightarrow \mathfrak{F}(G, \mathbb{R}),\left.\quad \bar{f} \mapsto \bar{f}\right|_{G}=f .
$$

Strictly monotone on edges functions on topological graphs were studied in connection with Kronrod-Reeb graphs of smooth functions on manifolds by V. V. Sharko in [2]. In that paper were given an existence criterion.

Definition 3.1. ([2, §3]) Let $\Gamma \in \Sigma$ be a connected topological graph. A func-orientation $\mathcal{O}$ of $\Gamma$ is an orientation on its edges having the following properties:

(1) $\Gamma$ has vertices of degree 1 with incoming and outcoming edges;

(2) each vertex of degree $\geqslant 2$ has an incoming and an outcoming edges;

(3) $\Gamma$ has no oriented closed cycles ${ }^{1}$.

Notice that func-orientation $\mathcal{O}$ of $\Gamma$ uniquely determines a partial order $P_{0}$ on vertices of $\Gamma: x<y$ iff there exists an oriented sequence of edges that starts in $x$ and ends in $y$.

Definition 3.2. $([2, \S 3])$ A partial order $P$ on the vertices of $\Gamma$ is said to be consistent with func-orientation $\mathcal{O}$ if the relations that are true for $P_{0}$ are also true for $P$. In other words, the identity map of the set of vertices is a morphism $P_{0} \rightarrow P$ of partially ordered sets.

Definition 3.3. $([2, \S 3])$ A func-oriented graph is a triple $(\Gamma, \mathcal{O}, P)$, where $\Gamma \in \Sigma, \mathcal{O}$ is a func-orientation of $\Gamma$ and $P$ is a partial order on vertices of $\Gamma$ which is consistent with the func-orientation $\mathcal{O}$.

\footnotetext{
${ }^{1} \mathrm{~A}$ set of oriented edges of $\Gamma$ that is homeomorphic to an oriented circle.
} 
In particular, if $\Gamma \in \Sigma$ admits func-orientation $\mathcal{O}$, then $\left(\Gamma, \mathcal{O}, P_{0}\right)$ is a func-oriented graph.

Also notice that every function $\bar{f} \in \mathfrak{M}(\bar{G}, \mathbb{R} \cup\{ \pm \infty\})$ determines a natural func-orientation $\mathcal{O}_{f}$ of edges towards increasing of $f$, and the consistent with it partial order $P_{f}$ on vertices given by the rule: $v_{1}<v_{2}$ if and only if $f\left(v_{1}\right)<f\left(v_{2}\right)$. In other words $f$ determines a func-oriented graph $\left(\bar{G}, \mathcal{O}_{f}, P_{f}\right)$.

Theorem 3.4. ([2, Theorem 3.1]) For each func-oriented graph $(\bar{G}, \mathcal{O}, P)$ there exists $f \in \mathfrak{M}(\bar{G}, \mathbb{R} \cup\{ \pm \infty\})$ such that $(\bar{G}, \mathcal{O}, P)=\left(\bar{G}, \mathcal{O}_{f}, P_{f}\right)$.

Since the assumption $f \in \mathfrak{F}(\bar{G}, \mathbb{R})$ is equivalent to $\bar{f} \in \mathfrak{M}(\bar{G}, \mathbb{R} \cup\{ \pm \infty\})$, we obtain the following:

Corollary 3.5. A graph $G \in \Theta$ admits a function $f \in \mathfrak{F}(G, \mathbb{R})$ iff $\bar{G}$ admits a func-orientation.

\section{Homotopies in Class $\mathfrak{F}(G, \mathbb{R})$}

Lemma 4.1. Let $G \in \Theta$ and $f, g \in \mathfrak{F}(G, \mathbb{R})$. Then the func-orientations induced by $f$ and $g$ on $\bar{G}$ coincide if and only if the functions $f$ and $g$ are homotopic in the class $\mathfrak{F}(G, \mathbb{R})$.

Proof. Necessity. Suppose $f$ and $g$ induce the same func-orientations on edges of $\bar{G}$ (and therefore of $G$ ). Then, for each vertex $v$ of $G$ its edges split into the same non-empty collections $A_{v}$ and $B_{v}$ such that

$$
\begin{array}{rlrl}
f\left(A_{v}\right) \subset(-\infty, f(v)), & & & f\left(B_{v}\right) \subset(f(v),+\infty), \\
g\left(A_{v}\right) \subset(-\infty, g(v)), & & g\left(B_{v}\right) \subset(g(v),+\infty) .
\end{array}
$$

Consider the following homotopy $h^{t}=t f+(1-t) g$ between $f$ and $g$ in the class of continuous functions. This formula easily implies that each $h^{t}$ is also monotone on each edge $e$, increases in the same direction as both $\left.f\right|_{e}$ and $\left.g\right|_{e}$, and defines the same splitting of edges incident to $v$ so that

$$
h^{t}\left(A_{v}\right) \subset\left(-\infty, h^{t}(v)\right), \quad h^{t}\left(B_{v}\right) \subset\left(h^{t}(v),+\infty\right) .
$$

Then by Lemma 2.1 each $h^{t}$ is open, whence that homotopy is in the class $\mathfrak{F}(G, \mathbb{R})$.

Sufficiency. Assume the functions $f$ and $g$ are homotopic in the class $\mathfrak{F}(G, \mathbb{R})$ by some homotopy $h^{t}$ but there exists an edge $e \in G$ and distinct points $x \neq y \in e$ such that $f(x)<f(y)$ and $g(x)>g(y)$. Then by continuity of $h$ there exists $t_{0} \in(0,1)$ such that $h^{t_{0}}(x)=h^{t_{0}}(y)$, whence $h^{t_{0}}$ is not strictly monotone on edges, and thus does not belong to $\mathfrak{F}(G, \mathbb{R})$. This gives a contradiction. 
This each $h^{t}$ induces certain orientation $\mathcal{O}_{t}$ of edges of $G$. By continuity of the homotopy those orientation must coincide for all $t \in[0,1]$. But $f=h^{0}$ and $g=h^{1}$, whence $\mathcal{O}_{f}=\mathcal{O}_{0}=\mathcal{O}_{1}=\mathcal{O}_{g}$.

Corollary 4.2. Let $G \in \Theta$. Then the homotopy classes of the set $\mathfrak{F}(G, \mathbb{R})$ in the class $\mathfrak{F}(G, \mathbb{R})$ are convex subsets of the linear space $C(G, \mathbb{R})$ of continuous functions on $G$.

\section{Counting homotopy Classes in $\mathfrak{F}(G, \mathbb{R})$}

Theorem 5.1. Let $G \in \Theta$ be a tree (i.e. has no cycles) consisting of $k$ vertices of degrees $c_{1}, \ldots, c_{k}$. Then the number of homotopy classes of $\mathfrak{F}(G, \mathbb{R})$ is

$$
2 \prod_{i=1}^{k}\left(2^{c_{i}-1}-1\right) .
$$

Proof. Since $G$ is a tree, it follows that $\bar{G}$ is also a tree, and therefore both such graphs admit func-orientations. Then by Corollary 3.5, the set $\mathfrak{F}(G, \mathbb{R})$ is non-empty. Moreover, due to Lemma 4.1, the number of path components of $\mathfrak{F}(G, \mathbb{R})$ is finite and is equal to the number of distinct orientations on edges of $G$ such that each vertex has incoming and outcoming edges. Let us calculate this number.

Choose a semifree edge $e$ with vertex $v_{1}$ of degree $c_{1}$. The map of $e$ can either preserve or reverse its orientation. Fix any orientation of $e$, say assume that $e$ is incoming for $v$. Then there are $2^{c_{1}-1}$ variants of orientation the remaining $c_{1}-1$ edges. All such variants are admissible except for the case when those $c_{1}-1$ edges are incoming for $v$ as well as $e$. Thus we obtain $2^{c_{1}-1}-1$ orientations of edges incident to $v$ assuming that one fixed edge $e$ is incoming.

Fix any admissible orientation at $v$ and consider the rest of the graph $G \backslash\left\{e_{1} \cup c_{1}\right\}$. It consists of $c_{1}-1$ trees for which of them we have have fixed an orientation of one semifree edges incident to $v_{1}$. Then applying the same arguments we will get that the total number of admissible orientation of $G$ with fixed orientation of $e_{1}$ is $\prod_{i=1}^{k}\left(2^{c_{i}-1}-1\right)$. Hence the total number of orientations $G$ is $2 \prod_{i=1}^{k}\left(2^{c_{i}-1}-1\right)$.

Theorem 5.2. Let $G \in \Theta$ be a func-orientable graph consisting of $k$ vertices of degrees $c_{1}, \ldots, c_{k}$ and has $m$ cycles. Denote by $N_{\mathfrak{F}(G, \mathbb{R})}$ the number of homotopy classes of functions of $\mathfrak{F}(G, \mathbb{R})$. Then

$$
\sup _{G^{\prime}} 2\left(\prod_{i=1}^{k}\left(2^{c_{i}^{\prime}-1}-1\right)\right) \leqslant N_{\mathfrak{F}(G, \mathbb{R})}<2\left(\prod_{i=1}^{k}\left(2^{c_{i}-1}-1\right)\right),
$$


where $G^{\prime}$ runs over all spanning subtrees (that is subtrees obtained from $G$ by removing $m$ edges), and $\left\{c_{i}^{\prime}\right\}$ are the degrees of vertices in $G^{\prime}$.

Proof. Let $G^{\prime}$ be any tree obtained by removing $m$ edges $e_{1}, \ldots, e_{m}$ from $G$. According to Theorem 5.1, the tree $G^{\prime}$ has $2 \prod_{i=1}^{k}\left(2^{c_{i}^{\prime}-1}-1\right)$ homotopy classes of maps from $\mathfrak{F}\left(G^{\prime}, \mathbb{R}\right)$ corresponding to distinct choices of orientations of its edges, where $c_{i}^{\prime}, i=1, \ldots, k$, are degrees of vertices in the tree $G^{\prime}$.

Evidently, every such an orientation of edges of $G^{\prime}$ extends to some orientation of edges of $G$, because the orientation for each removed edge $e_{i}$ is uniquely defined by values of both vertices of $e_{i}$ with a function that represents a homotopy class from $\mathfrak{F}\left(G^{\prime}, \mathbb{R}\right)$. Hence each homotopy class for the tree $G^{\prime}$ is also a homotopy class for $G$ which implies the inequality: $2\left(\prod_{i=1}^{k}\left(2^{c_{i}^{\prime}-1}-1\right)\right) \leqslant N_{\mathfrak{F}(G, \mathbb{R})}$.

Note that this inequality is not always strict. For example, the equality is reached on a graph that has exactly two homotopy classes of open functions and consists of two vertices $a$ and $b$, two edges incident to both $a$ and $b$, a semifree edge in $a$ and a semifree edge in $b$.

To prove the second inequality, instead of removing edges $e_{1}, \ldots, e_{m}$ from $G$ let us split each edge $e_{i}$ into two semifree edges by removing a point from its interior. This will give a new tree $G^{\prime \prime}$. By construction each homotopy class for $G$ is also a homotopy class for $G^{\prime \prime}$. This implies that $N_{\mathfrak{F}(G, \mathbb{R})}<2\left(\prod_{i=1}^{k}\left(2^{c_{i}-1}-1\right)\right)$. This inequality is in fact always strict, since there exist homotopy classes for $G^{\prime \prime}$ where the parts of split edges $e_{i}$ get different orientations which is impossible for $G$.

\section{REFERENCES}

[1] K. Kuratowski. Topology. Vol. I. New edition, revised and augmented. Translated from the French by J. Jaworowski. Academic Press, New York-London; Państwowe Wydawnictwo Naukowe, Warsaw, 1966.

[2] V. V. Sharko. About Kronrod-Reeb graph of a function on a manifold. Methods Funct. Anal. Topology, 12(4):389-396, 2006.

[3] И. А. Медных. О дискретных аналогах гиперэллиптических римановых поверхностей, pages 252-253. Казанское математическое общество, 2011 (in russian).

Received: June 16, 2020, accepted: September 12, 2020.

Igor Yu. Vlasenko

Topology laboratory, Department of Algebra and Topology, Institute of Mathematics of National Academy of Sciences of Ukraine, Tereschenkivska Str. 3, Kyiv, 01024, UkRAine

Email: vlasenko@imath.kiev.ua

ORCID: orcid.org/0000-0001-7870-9229 